\title{
Wie sich der Prämienanstieg weiter dämpfen lässt
}

\author{
Jürg Schlup \\ Dr. med., Präsident der FMH
}

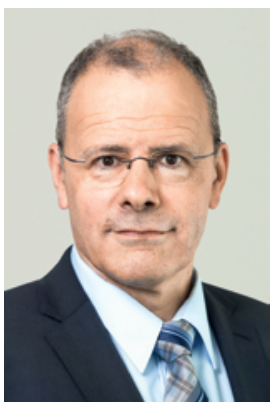

Communiqué santésuisse vom 12.7.2018. Gedämpftes Wachstum der Gesundheitskosten. URL: http://www.santesuisse.ch/de/details/ content/gedaempfteswachstum-der-gesundheitskosten

2 Medienmitteilung des Bundes vom 26.9.2016. Die Standardprämie steigt 2017 durchschnittlich um 4,5 Prozent. URL: https://www.admin.ch/ gov/de/start/dokumentation/medienmitteilungen.msg-id-63902.html

3 gfs.bern. Das Wichtigste in Kürze zum Gesundheitsmonitor 2018. Experimente unerwünscht, aber wachsende Ansprüche an die Versorgung. nterpharma, Basel, 2018

4 Verlauf von 1997 bis 2012 in «Wichtigstes in Kürze zum Gesundheitsmonitor 2012»; Verlauf 2010 bis 2016 in «Wichtigste in Kürze zum Gesundheitsmonitor 2016».

5 Golder et al. Verändertes Arbeitsumfeld und Einstellung zu neuen Finanzierungsmodellen tungsorientierung im Gesundheitswesen erkennbar. Schlussbericht von gfs.bern zur Begleitstudie im Auftrag der FMH. Januar 2018.

6 OBSAN. Analyse des International Health

Policy Survey (IHP). 2012 und 2015
Die diesjährige Prämiendiskussion ist mit ungewöhnlich vielen positiven Nachrichten verbunden: Die Kosten der obligatorischen Krankenpflegeversicherung (OKP) stiegen im Laufe des Jahres 2017 mit 1,7\% nicht nur deutlich weniger an als im langjährigen Durchschnitt [1] - der Kostenanstieg fiel damit auch viel geringer aus als die Prämienerhöhung von 4,5\%, die für 2017 vorgenommen wurde [2].

Eine weitere gute Nachricht ist, dass mit 87\% der Bevölkerung so viele Menschen wie noch nie zuvor unser Gesundheitswesen positiv bewerten [3]. Gleichzeitig haben seit der Einführung des KVG [4] noch nie weniger Personen die Krankenkassenprämien als dauerhaftes (5\%) oder gelegentliches (11\%) Problem bezeichnet als heute [3]. Noch im Jahr 2010 waren es mehr als dreimal so viele.

Dennoch dürfen unsere Anstrengungen für stetige Verbesserungen eines qualitativ hochstehenden Gesundheitswesens zu möglichst geringen Kosten nicht nachlassen. Dabei sind nach Auffassung der FMH drei zentrale Stossrichtungen zu verfolgen. Erstens braucht es als entscheidende Weichenstellung die einheitliche Finanzierung ambulanter und stationärer Leistungen (EFAS). Nur so können Behandlungen zunehmend ambulant und folglich kostengünstiger durchgeführt werden, ohne den Prämienzahler stärker zu belasten. Zudem würde die integrierte Versorgung gestärkt und damit ein grosses Sparpotential realisiert. Zweitens erfordert die stark vorangetriebene Verlagerung von stationär nach ambulant rasche Anpassungen unserer Spitalstrukturen wie überkantonale Spitalversorgungsplanung und ambulante OP-Zentren.

Unverzichtbare Voraussetzung einer solchen Entwicklung ist die Lösung der Interessenkonflikte der Kantone, deren Mehrfachrolle als Spitalplaner und -eigentümer, als Auftraggeber und Finanzierer sowie als Aufsichts- und Tarifinstanz andernfalls Fehlkapazitäten zementiert.

Drittens bietet die überbordende administrative Belastung grosses Sparpotential. Ein Spitalarzt der Akutsomatik verbringt heute knapp ein Drittel seiner Ar- beitszeit mit Administration [5]. Alleine die Zunahme des Aufwands für Patientendossiers seit 2011 erfordert jedes Jahr 100 neue ärztliche Vollzeitstellen in unserem Land. Auch bei den praktizierenden Ärztinnen und Ärzten wächst die administrative Belastung [6]. Wie Ärztinnen und Ärzte konstruktiv an Verbesserungen arbeiten, zeigt der VSAO auf S. 1097 in dieser Ausgabe auf. Die nun lancierte zweite Welle der VSAOKampagne "Medizin statt Bürokratie» will dieses wichtige Anliegen zudem in die Bundespolitik tragen.

\section{7 stiegen die Kosten der obligatorischen} Krankenpflegeversicherung (OKP) um 1,7\% deutlich weniger als die Standardprämie mit $4,5 \%$.

Dass dies gelingt, ist für eine kosteneffiziente Weiterentwicklung unseres Gesundheitswesens zentral, denn bislang wird das Problem der administrativen Belastung auf Bundesebene kaum berücksichtigt. So findet sich das Wort "administrativ» im 131-seitigen Expertenbericht zur Kostendämpfung lediglich sieben Mal - obwohl einige seiner Massnahmen eine weitere Zunahme der Bürokratie erwarten lassen. Der Blick ins Ausland zeigt z.B., dass die Einführung eines Globalbudgets den Ärzten noch mehr Administration aufbürdet und grosse Verwaltungsstrukturen erfordert.

\section{Kostendämpfung braucht einheitliche Finan-} zierung, überkantonale Spitalversorgung und den Abbau administrativer Belastung.

Ob hier wachsende Administration mehr Nutzen als Kosten verursacht, darf bezweifelt werden. Für die $\mathrm{Pa}$ tientenversorgung hingegen weisen Studien darauf hin, «dass die heutige Gesundheitsversorgung den Versicherten insgesamt mehr Nutzen generiert, als sie an Krankenkassenprämien kostet», wie der Gesundheitsökonom Harry Telser in seinem Artikel auf S. 1094 dieser Ausgabe erläutert. Auch dies ist eine positive Nachricht zur Prämiendiskussion. 